\title{
Effect of including damage at the tissue level in the nonlinear homogenisation of trabecular bone
}

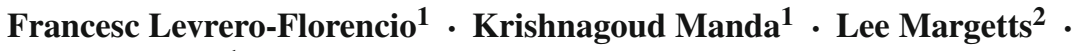 \\ Pankaj Pankaj $^{1}$
}

Received: 24 August 2016 / Accepted: 21 April 2017 / Published online: 12 May 2017

(C) The Author(s) 2017. This article is an open access publication

\begin{abstract}
Being able to predict bone fracture or implant stability needs a proper constitutive model of trabecular bone at the macroscale in multiaxial, non-monotonic loading modes. Its macroscopic damage behaviour has been investigated experimentally in the past, mostly with the restriction of uniaxial cyclic loading experiments for different samples, which does not allow for the investigation of several load cases in the same sample as damage in one direction may affect the behaviour in other directions. Homogenised finite element models of whole bones have the potential to assess complicated scenarios and thus improve clinical predictions. The aim of this study is to use a homogenisation-based multiscale procedure to upscale the damage behaviour of bone from an assumed solid phase constitutive law and investigate its multiaxial behaviour for the first time. Twelve cubic specimens were each submitted to nine proportional strain histories by using a parallel code developed in-house. Evolution of post-elastic properties for trabecular bone was assessed for a small range of macroscopic plastic strains in these nine load cases. Damage evolution was found to be non-isotropic, and both damage and hardening were found to depend on the loading mode (tensile, compression or shear); both were characterised by linear laws with relatively high coefficients of determination. It is expected that the knowledge of the macroscopic behaviour of trabecular bone gained in this study will help in creating more precise continuum FE models of whole bones that improve clinical predictions.
\end{abstract}

$凶$ Francesc Levrero-Florencio

f.levrero-florencio@ed.ac.uk

1 School of Engineering, The University of Edinburgh, The King's Buildings, Edinburgh EH9 3DW, UK

2 School of Mechanical, Aerospace and Civil Engineering, The University of Manchester, Sackville Street, Manchester M13 OPL, UK
Keywords Trabecular bone - Multiscale modelling · Plasticity $\cdot$ Damage $\cdot$ Finite element method

\section{Introduction}

Bone is a hierarchical biomaterial, exhibiting complex postyield properties at each of its scales (Schwiedrzik et al. 2013). The mechanical behaviour of trabecular bone at the macroscale is often modelled by using homogeneous isotropic linear elasticity, with separate sets of elastic constants being assigned to cortical and trabecular bone (Completo et al. 2009; Conlisk et al. 2015). Site-specific mineral heterogeneity at the macroscale is often included by using computed tomography (CT) scans, which allow for variation of properties on the basis of CT attenuations (Helgason et al. 2008; Schileo et al. 2008; Tassani et al. 2011). However, bone mineral density alone is not enough to accurately predict the stiffness of trabecular bone at the macroscale since it is known to be anisotropic, mostly because of its heavily directional microstructure (Odgaard et al. 1997; Turner et al. 1990).

The macroscopic stiffness tensor of trabecular bone has been evaluated by using micro-CT $(\mu \mathrm{CT})$ and homogenisation-based multiscale finite element (FE) models, the socalled micro-FE ( $\mu \mathrm{FE}$ ) models (Hollister et al. 1994; van Rietbergen et al. 1995). This method consists of picking a cubic region and converting the binarised $\mu \mathrm{CT}$ scans to high resolution $\mathrm{FE}$ meshes, which include detailed geometry of bone microstructure. The solid phase is usually assigned isotropic elastic properties, and the cubic specimen is then subjected to six virtual load cases, stress or strain (Hollister and Kikuchi 1992). The response from these tests enables the evaluation of the macroscopic elastic stiffness tensor by using a standard mechanics approach (van Rietbergen et al. 1996). 
This methodology has been extensively used, and studies have also established relationships between these stiffness tensors and the micro-architectural indices of the considered samples, specifically bone volume over total volume fraction (BV/TV) and fabric tensor (Cowin 1985; Zysset and Curnier 1995; Zysset 2003).

This approach has been extended to predict the yield criterion of trabecular bone (Cowin 1986; Bayraktar and Keaveny 2004; Wolfram et al. 2012; Sanyal et al. 2015; LevreroFlorencio et al. 2016). These studies use FE meshes derived from $\mu \mathrm{CT}$ scans which are subjected to multiple load cases to evaluate the homogenised yield surface. The $0.2 \%$ criterion is then used to assess where the yield point is located, which means that the elastic slope intercepts the $X$-axis at a value of $0.2 \%$ macroscopic strain. In a multiaxial context, the macroscopic yield point is located where the macroscopic elastic slope intercepts the macroscopic stress norm-macroscopic strain norm curve. Isotropic elastoplastic assumption is made for the constitutive law at the solid phase level. Some of these studies used an asymmetric principal strain-based criterion to represent the onset of yield (Bayraktar and Keaveny 2004; Wolfram et al. 2012; Sanyal et al. 2012), but others used an approximation to a Drucker-Prager yield surface (Panyasantisuk et al. 2015; Levrero-Florencio et al. 2016), as suggested by Tai et al. (2006) and Carnelli et al. (2010).

Nanoindentation experiments on bone suggest that its solid phase can be effectively modelled by using a pressuredependent yield surface, arising from its cohesive-frictional behaviour (Tai et al. 2006). Therefore, it has been suggested that the solid phase of bone can be modelled using classical yield surfaces such as Mohr-Coulomb or DruckerPrager (Carnelli et al. 2010; Tai et al. 2006). The resulting macroscopic yield criteria have been defined using both stress and strain-based descriptions (Keaveny et al. 1994; Keller 1994; Kopperdahl and Keaveny 1998). Studies have shown that the yield surface in strain space is approximately isotropic and independent of BV/TV (Bayraktar and Keaveny 2004; Levrero-Florencio et al. 2016) and consequently easy to apply in macroscopic models (Pankaj 2013; Pankaj and Donaldson 2013). Maghous et al. (2009) showed that the macroscopic yield surface of a porous material with a Drucker-Prager yield surface for the matrix material reduces to an eccentric ellipsoid, with the corresponding decrease in uniaxial strength values due to the presence of porosity. This suggests that trabecular bone at the macroscale can be modelled with an eccentric ellipsoid, or Tsai-Wu, which has been shown by Cowin (1986), Wolfram et al. (2012), Panyasantisuk et al. (2015) and Levrero-Florencio et al. (2016).

After yield, there is little information on how the macroscopic response of trabecular bone evolves with further loading; hardening is usually assumed to be isotropic in computational models (Garcia et al. 2009; Schwiedrzik and
Zysset 2013). It is not possible to experimentally test different load directions after yield in the same sample since samples tested once cannot be retested as damage in one direction may affect the rest of directions and finding two or more samples with highly resembling microstructure is not possible. This makes it impossible to experimentally obtain the macroscopic multiaxial post-yield behaviour of trabecular bone. The $\mu \mathrm{FE}$ approach again presents an opportunity to understand this via computational means. To evaluate the macroscopic post-yield response, once again, the solid phase constitutive model needs to be provided. Bone shows two main mechanisms of energy dissipation after yield: plastic deformation and elastic stiffness reduction, or damage (Schwiedrzik and Zysset 2013). With regard to hardening of the solid phase, it has been assumed to be linear, with a slope of 5\% its elastic stiffness, in previous homogenisation studies (Bayraktar and Keaveny 2004; Wolfram et al. 2012). A recent study showed that the hardening of the extracellular matrix, which can be considered to be a scale below the solid phase of trabecular bone, is, however, slightly nonlinear (Schwiedrzik et al. 2014).

Damage behaviour of bone at different scales has been studied and modelled in several studies (Keaveny et al. 1999; Schwiedrzik and Zysset 2013; Garcia et al. 2009). Garcia et al. (2009) developed a macroscopic constitutive model for bone- a yield surface defined in stress space using the fabric-based elastic compliance tensor and a damage threshold modelled with a halfspacewise generalisation of the Hill criterion (Rincón-Kohli and Zysset 2009). Schwiedrzik and Zysset (2013) developed a constitutive model for bone which is potentially applicable to different length scales, ranging from the ultrastructural to the macroscopic level. It includes anisotropic elasticity based on a multiscale homogenisation scheme proposed by Reisinger et al. (2010), an eccentric elliptical surface which describes the onset of yield and damage (Wolfram et al. 2012; Levrero-Florencio et al. 2016), and viscoplasticity described by a Perzyna formulation (Ponthot 1998). The damage variable used in these two above-cited studies is scalar and thus describes isotropic damage evolution, i.e. damage equally affects all directions. While this presents a relatively simple model, it appears unlikely that damage due to loading in one direction will affect stiffness components, isotropically, in all directions.

This study uses a $\mu$ FE-based nonlinear homogenisation approach, with plasticity and damage, to evaluate how assumptions made for the solid phase of trabecular bone affect its macroscopic post-yield behaviour. The first aim of this study is to assess how the macroscopic stiffness components are affected by the initiation and development of microscopic damage. The second aim is to assess how macroscopic damage is related to the macroscopic strain norm and how well it can be predicted for different load cases. The 
third aim is to assess the evolution of the macroscopic yield surface, in both strain and stress space.

\section{Materials and methods}

\subsection{Experimental samples and imaging procedure}

Fresh bovine proximal femurs ( $<2.5$ years old $)$ were obtained from a local abattoir and were stored at $-20^{\circ} \mathrm{C}$ until they were cored. Ten cores were extracted from the trochanteric region of these femurs. Diamond-coated coring tools (Starlite Industries, Rosemont PA, USA) were used to extract the specimens, and then the top and bottom faces of the cylinders were cut with a slow-speed saw (Isomet 1000, Buehler, Düsseldorf, Germany). $\mu \mathrm{CT}$ scans were taken for each specimen by using a Skyscan $1172 \mu \mathrm{CT}$ scanner (Bruker, Kontich, Belgium) at a resolution of $17.22 \mu \mathrm{m}$. The scanning parameters were $94 \mathrm{kV}, 136 \mathrm{~mA}$ and $200 \mathrm{~ms}$ integration time with four scans taken in 720 equiangular positions. The grey-scale images were binarised with a thresholding script that does not require user intervention (Gómez et al. 2013).

Twelve 5-mm virtual cubes were extracted from the binarised cylinders. This volume element (VE) size has been previously used and therefore is considered to be appropriate to capture the features of trabecular bone (Harrigan et al. 1988; van Rietbergen et al. 1995; Sanyal et al. 2015). The mean intercept length (MIL) fabric tensor (Harrigan and Mann 1984) was evaluated using BoneJ (Doube et al. 2010) and then used to align the images with the fabric. This approach has been used in previous studies (Wolfram et al. 2012; Levrero-Florencio et al. 2016). The alignment of the cubes was rechecked after the $5 \mathrm{~mm}$ cropping to ensure that the misalignment was smaller than $8^{\circ}$ (Wolfram et al. 2012; Sanyal et al. 2015; Levrero-Florencio et al. 2016). The $\mathrm{BV} / \mathrm{TV}$ of the samples ranged from 14.8 to $30.3 \%$, and their degree of anisotropy (DOA) ranged from 1.61 to 3.47.

\subsection{Solid phase constitutive model}

The mathematical operators defined in this section largely follow the notation used in Schwiedrzik et al. (2013), Panyasantisuk et al. (2015) and Levrero-Florencio et al. (2016). We have used tensor notation, which can be alternatively expressed using index notation as shown within the parentheses in the following. For example, a second-order tensor and its representation in index notation are: $\mathbf{A}\left(A_{i j}\right)$. A double contraction of a second-order tensor and a fourth-order tensor is expressed as $\mathbf{A}: \mathbb{B}\left(A_{i j} \mathbb{B}_{i j k l}\right)$; a double contraction of a fourth-order tensor and a second-order tensor is expressed as $\mathbb{A}: \mathbf{B}\left(\mathbb{A}_{i j k l} B_{k l}\right)$; a double contraction of two second-order tensors is expressed as $\mathbf{A}: \mathbf{B}\left(A_{i j} B_{i j}\right)$; a tensor product between two second-order tensors is expressed as $\mathbf{A} \otimes \mathbf{B}\left(A_{i j} B_{k l}\right)$; and a symmetric tensor product between two second-order tensors is expressed as $\mathbf{A} \underline{\bar{\otimes}} \mathbf{B}\left(\frac{1}{2}\left[A_{i k} B_{j l}+\right.\right.$ $\left.A_{i l} B_{j k}\right]$ ).

The elastic regime of the solid phase was defined as an isotropic linear material, by using Hencky's hyperelasticity (de Souza Neto et al. 2008), with a Young's Modulus of 12,700 MPa and a Poisson's ratio of 0.3 (Wolfram et al. 2012; Levrero-Florencio et al. 2016). As Cowin (1997) stated, there is little to no error in assuming tissue isotropy for trabecular bone. A quadric yield surface was used to describe the onset of yield and damage of the solid phase (Schwiedrzik et al. 2013; Schwiedrzik and Zysset 2015). The yield function $f$ was defined as

$f(\boldsymbol{\sigma})=\sqrt{\sigma: \mathbb{F}: \sigma}+\mathbf{F}: \sigma-R\left(\bar{\varepsilon}^{p}\right)=0$,

where $\sigma$ is the stress, $\mathbb{F}$ and $\mathbf{F}$ are, respectively, fourth- and second-order tensors which define the shape and eccentricity of the yield surface, $R$ is the radius of the isotropic yield criterion, and $\bar{\varepsilon}^{p}$ is the accumulated plastic strain. Note that the yield surface is defined in stress space, and not in effective stress space (Schwiedrzik and Zysset 2015).

The fourth-order tensor $\mathbb{F}$ and the second-order tensor $\mathbf{F}$ are defined as

$\mathbb{F}=-\zeta_{0} F_{0}^{2}(\mathbf{I} \otimes \mathbf{I})+\left(\zeta_{0}+1\right) F_{0}^{2}(\mathbf{I} \underline{\bar{\otimes}} \mathbf{I})$

and

$\mathbf{F}=\frac{1}{2}\left(\frac{1}{\sigma_{0}^{+}}-\frac{1}{\sigma_{0}^{-}}\right) \mathbf{I}$,

where

$F_{0}=\frac{\sigma_{0}^{+}+\sigma_{0}^{-}}{2 \sigma_{0}^{+} \sigma_{0}^{-}}$

Equation 1 approximates a Drucker-Prager criterion when $\zeta_{0}=0.49$. Recent nanoindentation studies on bone tissue suggest that a Mohr-Coulomb or a Drucker-Prager surface could approximate the yield criterion at the microscopic level (Tai et al. 2006; Carnelli et al. 2010) and it has been recently used in homogenisation studies (Panyasantisuk et al. 2015; Levrero-Florencio et al. 2016). The uniaxial yield strains for use in the criterion were assumed to be $0.41 \%$ in tension and $0.83 \%$ in compression (Bayraktar and Keaveny 2004) and were then converted to yield stresses by using the procedure described in Schwiedrzik et al. (2016). This means that the corresponding yield stresses are $E_{0} \varepsilon_{0}^{+}=52 \mathrm{MPa}$ for tension and $E_{0} \varepsilon_{0}^{-}=105 \mathrm{MPa}$ for compression, where $E_{0}$ is the undamaged Young's modulus. Linear isotropic hardening of $5 \%$ of the elastic slope was also assumed (Wolfram et al. 2012; Sanyal et al. 2015; Panyasantisuk et al. 2015). 
Isotropic damage evolution was assumed to be coupled with plasticity, exactly as in Schwiedrzik and Zysset (2013, 2015), and thus it is defined as

$D\left(\bar{\varepsilon}^{p}\right)=D_{c}\left(1-\mathrm{e}^{-k_{p} \bar{\varepsilon}^{p}}\right)$,

where $D_{c}$ is the maximum damage, which is capped at 0.9 to avoid numerical difficulties related to the complete loss of stress carrying capacity in any region of the model; the inverse damage rate $1 / k_{p}$ was set to $9.53 \%$ (Schwiedrzik and Zysset 2013). Implementation of the model in an implicit FE context is described in "Appendix".

\subsection{Computational procedure}

The twelve VEs were meshed with trilinear hexahedra, by converting every voxel of the binarised CT scans to a FE element. The largest obtained mesh had around nine million nodes and thus around 27 million degrees of freedom.

Each of the VEs was subjected to nine strain-controlled uniaxial cases: three tensile, three compressive and three shear cases. Kinematic uniform boundary conditions were used to constrain the VEs, which were applied as described by Wang et al. (2009). According to Wang et al. (2009) and Panyasantisuk et al. (2015), these boundary conditions (BC) provide an upper bound for the macroscopic stiffness tensor and macroscopic yield surface of trabecular bone.

FE simulations were run on a Cray XC30 supercomputer hosted by ARCHER (UK National Supercomputing Service). The used software was an in-house parallel implicit finite strain FE solver, developed within the context of ParaFEM (Margetts 2002; Smith et al. 2013), which uses Message Passing Interface (MPI) to perform the parallelisation (The MPI Forum 1993). Each simulation took from 40 to 100 min when using 1920 cores, depending on the load case, with compression load cases taking the longest. The initial step size corresponded to $0.1 \%$ macroscopic strain norm and was permitted to decrease to a minimum of $0.001 \%$ if global or local convergence was not achieved in larger increments.

In order to enlarge the region of convergence of the Newton-Closest-Point Projection Method (Newton-CPPM) scheme, a line search procedure was implemented as in the primal-CPPM algorithm proposed by Pérez-Foguet and Armero (2002). To ensure that a possible fail of convergence of the CPPM scheme does not influence the results of our FE simulation, lack of convergence of the CPPM at any integration point is broadcasted to all MPI processes in order to cut down the time increment to half of its value. A Newton-Raphson scheme was used as the global solution tracking procedure, and a preconditioned conjugate gradient method was used as the linear algebraic solver.

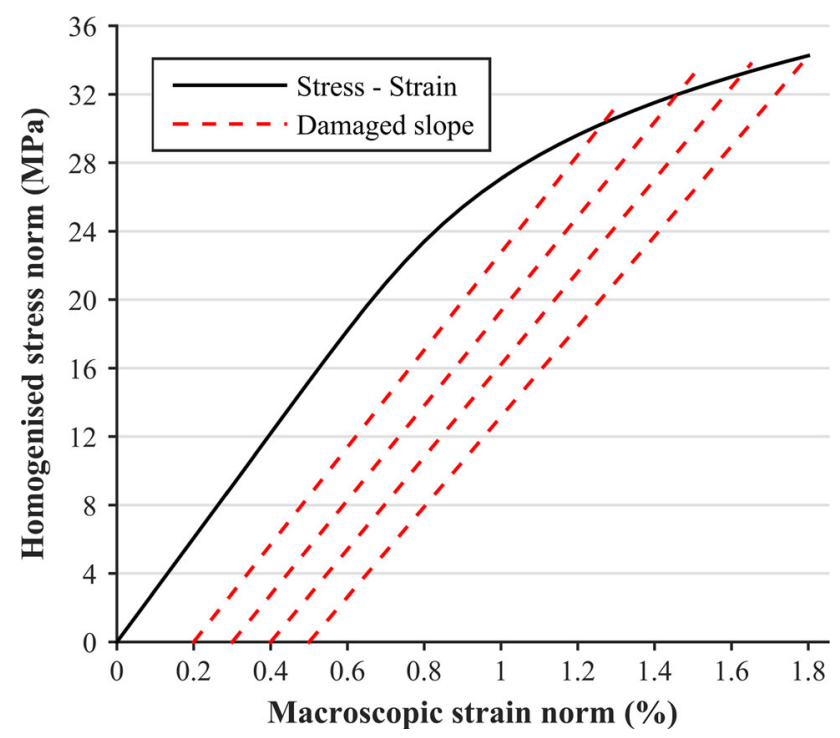

Fig. 1 Definition of the macroscopic strain points with the damaged slopes; this is the compression case in direction 1 (i.e. $-\varepsilon_{11}$ ) for the densest sample $(\mathrm{BV} / \mathrm{TV}=30.3 \%)$. The slope at $0.5 \%$ macroscopic strain norm is approximately $12 \%$ lower than the undamaged slope

\subsection{Definition of the macroscopic strain points}

The yield points were described in the homogenised GreenLagrange strain norm-homogenised Second Piola-Kirchhoff stress norm plane (Fig. 1). The homogenised stress is then defined as

$\boldsymbol{\sigma}_{\text {hom }}=\frac{1}{V_{0}} \sum_{i=1}^{\text {nel }} \sum_{j=1}^{\text {nip }} w_{i} \operatorname{det}\left(\mathbf{J}_{i j}\right) \boldsymbol{\sigma}_{i j}$,

where no summation is implied over repeated indices, $V_{0}$ is the initial volume of the VE, nel is the number of elements in the system, nip is the number of integration points in a trilinear hexahedron, $w_{i}$ are the weights corresponding to a trilinear hexahedron, $\mathbf{J}$ is the Jacobian, and $\boldsymbol{\sigma}$ is the stress at the solid phase level. Note that due to the relatively small yield strains, an infinitesimal strain formulation can be used at the macroscale (Wolfram et al. 2012; Schwiedrzik et al. 2014).

The homogenised orthotropic elastic stiffness tensor was calculated at every time increment using the procedure described by van Rietbergen et al. (1995, 1996), and the damage variable was used to reduce the integration point-specific solid phase stiffness tensor. Since the sample was already aligned according to the directions described by the MIL fabric tensor, the resulting elasticity tensor was assumed to be orthotropic and aligned with the MIL axes (Odgaard et al. 1997). The $0.2 \%$ criterion was used to define the yield points (Wolfram et al. 2012) and extended to further define additional strain points at $0.3,0.4$ and $0.5 \%$ by using the procedure shown in Fig. 1. These points will henceforth be referred to 
as macroscopic strain norms (e.g. $0.2 \%$ macroscopic strain norm). Note that if yield is considered to occur at $0.2 \%$, the following points $(0.3,0.4$ and $0.5 \%)$ could be considered as $0.1,0.2$ and $0.3 \%$ macroscopic plastic strain norms, respectively (with damaged slope). Clearly, these points defined with the damage will correspond to larger macroscopic total strains in comparison with those evaluated without damage. The appropriate damaged slope to define the strain points is calculated for the corresponding load case at each time step, by using the damaged macroscopic stiffness tensor. An example is presented here for a strain-controlled uniaxial case in direction 1.

In the following, the homogenised stress is the projection of the macroscopic elastic strain through the macroscopic damaged stiffness tensor, the first subscript denotes a label, and the following subscripts denote indices of the tensor. Consider application of a normal strain in direction 1, the elastic system can be written in indicial notation as

$\sigma_{\mathrm{hom}, i j}=D_{\mathrm{dam}, i j 11} \epsilon_{0,11}^{e}$,

where $D_{\mathrm{dam}, i j k l}$ are the components of the damaged macroscopic stiffness tensor. When the norm of the corresponding homogenised stress is calculated, the following expression can be derived, by taking into account the orthotropy of the macroscopic stiffness tensor,

$$
\begin{aligned}
& \left\|D_{\mathrm{dam}, i j 11} \epsilon_{0,11}^{e}\right\| \\
& \quad=\sqrt{D_{\mathrm{dam}, 1111}^{2} \epsilon_{0,11}^{e, 2}+D_{\mathrm{dam}, 2211}^{2} \epsilon_{0,11}^{e, 2}+D_{\mathrm{dam}, 3311}^{2} \epsilon_{0,11}^{e, 2}},
\end{aligned}
$$

and thus the damaged slope used to calculate the macroscopic strain points can be expressed as

$$
K_{\mathrm{dam}}=\sqrt{\left(D_{\mathrm{dam}, 1111}^{2}+D_{\mathrm{dam}, 2211}^{2}+D_{\mathrm{dam}, 3311}^{2}\right)} .
$$

\section{Results}

\subsection{Stiffness reduction}

The damaged orthotropic stiffness components $\left(E_{11}, E_{22}\right.$, $E_{33}, G_{12}, G_{13}$ and $G_{23}$ ) are obtained from the damaged macroscopic stiffness tensor. These are then normalised by dividing them by the corresponding undamaged orthotropic stiffness and plotted for every sample and for every considered load case (Fig. 2). Figure 2 shows that in spite of isotropic damage being assumed at the solid phase level, its effect on the macroscopic level is not isotropic, and that its effect depends on the considered loading mode (i.e. tension, compression or shear). It can be seen that while all stiffness components reduce in all load cases, the stiffness component corresponding to the load case the sample is subjected to reduces the most. It is also interesting to note that in the case of a strain-controlled uniaxial normal load case, the shear stiffness components corresponding to the shear planes containing the loaded normal component reduce more than the other one (e.g. if the normal case is in direction $1, G_{12}$ and $G_{13}$ reduce more than $G_{23}$ ).

Stiffness reductions for each of the considered load cases were related to BV/TV, corresponding initial orthotropic stiffness component, and macroscopic strain norm through multilinear regression analyses. Only relationships with respect to macroscopic strain norms were found to be significant $(p<0.05)$. Therefore, we re-evaluated these multilinear regressions as linear regressions, only with respect to macroscopic strain norms $(p \rightarrow 0)$. The coefficients of determination $\left(R^{2}\right)$, the intercepts and the slopes of these fits are shown in Table 1. Figure 3 illustrates these fits along with the actual data points. It can be seen from this figure that damage development under strain-controlled uniaxial tension and strain-controlled uniaxial compression can be reasonably well predicted by a linear relationship with respect to the macroscopic strain norm, but not so well for shear, as the coefficients of determination suggest. It is also important to point out that for the considered range of post-elastic strains, damage development can be reasonably well approximated with a line although the relationship between damage and accumulated plastic strain at the solid phase is exponential (Eq. 5).

Figure 4 shows the stiffness reduction for the most porous and the densest samples, for the load case in which a straincontrolled uniaxial tension is applied in direction 1, for different macroscopic strain norm levels. As expected, the decrease in stiffness increases with increasing strain norm, a trend which was true for all the samples. For the samples shown, it can also be seen that in the denser sample the difference in stiffness reduction between the stiffness component corresponding to the load case the sample is subjected to and the others is smaller than for the porous sample. Therefore, we considered linear relationship between BV/TV and the difference between the stiffness reduction in the component corresponding to the load case the sample is subjected to and the average of the rest of stiffness components. However, poor statistical significance was found $(p>0.05)$, indicating that this was not a general trend.

\subsection{Hardening of the macroscopic yield surface}

Macroscopic yield stresses have been related to fabric and $\mathrm{BV} / \mathrm{TV}$ in previous studies; however, relationships between macroscopic yield strains and BV/TV are weak and relationships between macroscopic yield strains and fabric are moderate, but significant (Wolfram et al. 2012; Panyasan- 

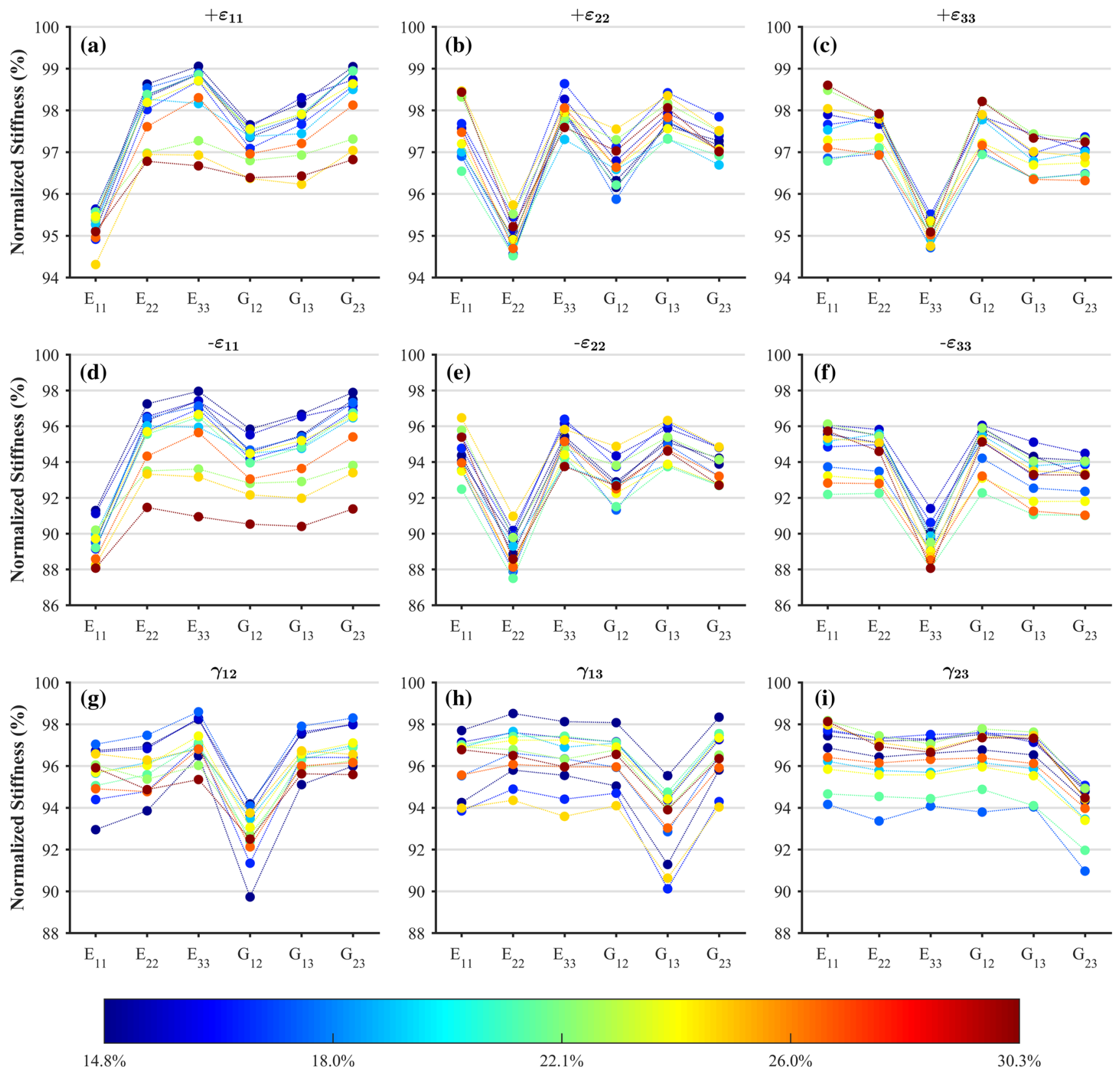

Fig. 2 Normalised orthotropic stiffness for all the samples, and for all the considered strain-controlled uniaxial load cases: tensile loading in direction $1(\mathbf{a}), 2(\mathbf{b})$ and $3(\mathbf{c})\left(+\varepsilon_{11},+\varepsilon_{22}\right.$ and $+\varepsilon_{33}$, respectively); compressive loading in direction $1(\mathbf{d}), 2(\mathbf{e})$ and $3(\mathbf{f})\left(-\varepsilon_{11},-\varepsilon_{22}\right.$ and $-\varepsilon_{33}$, respectively); and shear loading in plane $1-2(\mathbf{g}), 1-3(\mathbf{h})$ and $2-$ 3(i) $\left(\gamma_{12}, \gamma_{13}\right.$ and $\gamma_{23}$, respectively). The colour coding is on the basis of BV/TV and is used as a labelling mechanism. The points defined at $0.5 \%$ macroscopic strain norm have been considered in this figure

Table 1 Values of the coefficients of determination $R^{2}$, intercepts and slopes for the linear fits between damage and macroscopic strain norms, for each of the considered load cases

\begin{tabular}{llllllllll}
\hline Load case & $+\epsilon_{11}$ & $+\epsilon_{22}$ & $+\epsilon_{33}$ & $-\epsilon_{11}$ & $-\epsilon_{22}$ & $-\epsilon_{33}$ & $\gamma_{12}$ & $\gamma_{13}$ & $\gamma_{23}$ \\
$R^{2}$ & 0.92 & 0.91 & 0.95 & 0.87 & 0.84 & 0.85 & 0.57 & 0.37 & 0.60 \\
Intercept (\%) & 0.66 & 0.66 & 0.52 & 1.96 & 2.37 & 1.89 & 1.22 & 1.45 \\
Slope (\%) & 832.7 & 874.4 & 880.0 & 1717.5 & 1726.6 & 1757.6 & 1211.3 & 1061.6 & 1101.5 \\
\hline
\end{tabular}



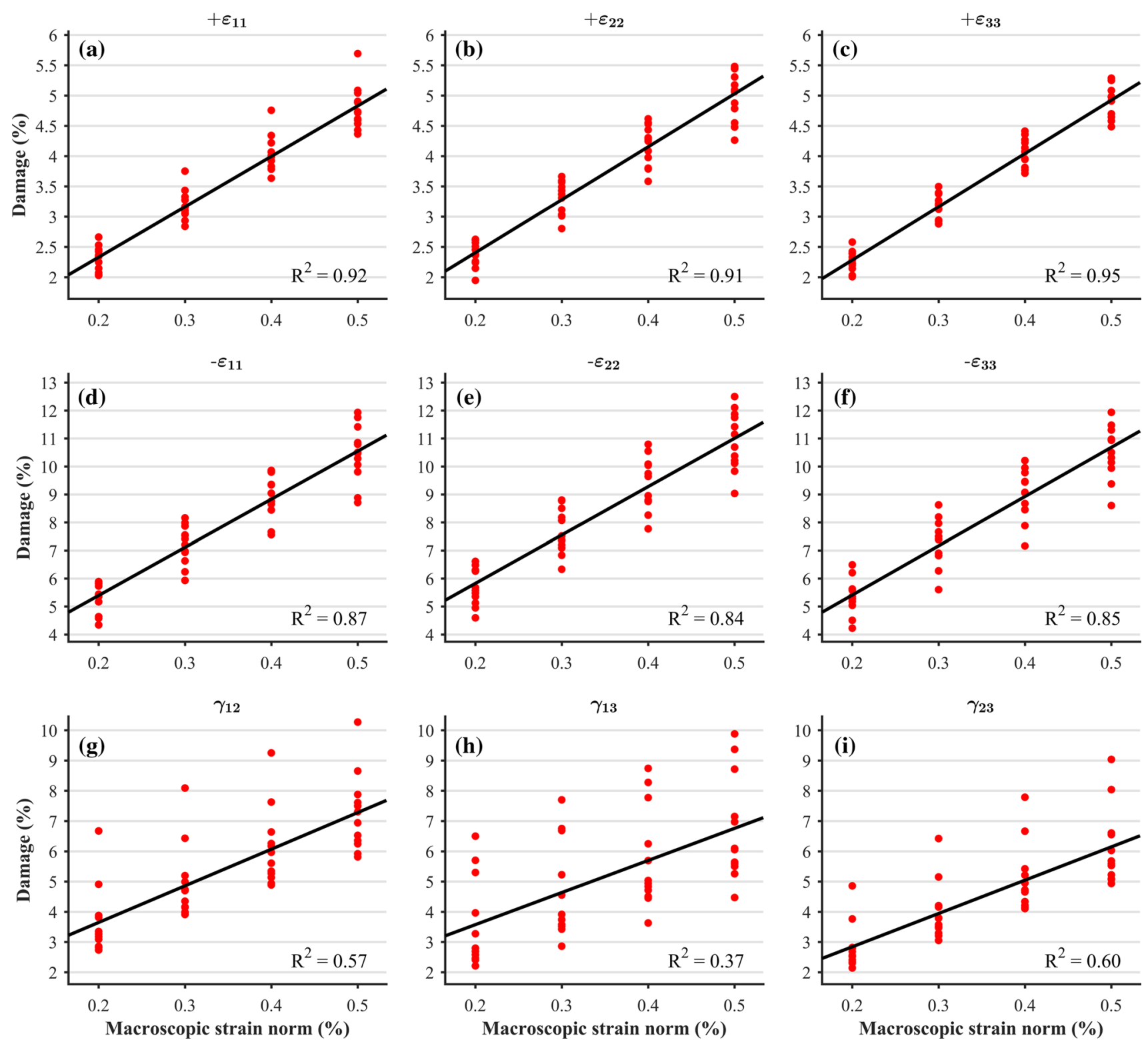

Fig. 3 Linear fits between damage and macroscopic strain norms, and the corresponding data points. The considered uniaxial strain-controlled load cases are: tensile loading in direction $1(\mathbf{a}), 2(\mathbf{b})$ and $3(\mathbf{c})\left(+\varepsilon_{11}\right.$,

$+\varepsilon_{22}$ and $+\varepsilon_{33}$, respectively); compressive loading in direction $1(\mathbf{d})$, 2 (e) and $3(\mathbf{f})\left(-\varepsilon_{11},-\varepsilon_{22}\right.$ and $-\varepsilon_{33}$, respectively); and shear loading in plane $1-2(\mathbf{g}), 1-3(\mathbf{h})$ and $2-3(\mathbf{i})\left(\gamma_{12}, \gamma_{13}\right.$ and $\gamma_{23}$, respectively)

tisuk et al. 2015). Also, as previously stated, the orthotropic stiffness components have been related to these microarchitectural indices (Odgaard et al. 1997; Zysset 2003). Therefore, we considered inclusion of orthotropic stiffness components in our regressions for the yield stresses (rather than yield strains). Macroscopic yield stress norms were related to the corresponding initial orthotropic stiffness and the macroscopic strain norms through multilinear regressions. These fits and the corresponding data points are shown in Fig. 5. It can be seen that higher yield stress results from higher initial stiffness and from the choice of higher macro- scopic strain norm. The coefficients of determination and slopes of these fits are shown in Table 2. All of these fits were found to be statistically significant $(p<0.05)$.

Macroscopic yield strain norms were related to the macroscopic strain norms through linear regressions. These fits and the corresponding data points are shown in Fig. 6. The coefficients of determination and slopes of these fits are provided in Table 3. All of these fits were statistically significant $(p<0.05)$. In general, it is found that hardening in both stress and strain spaces depends on the loading mode, i.e. tension, compression or shear, but it is not anisotropic. 


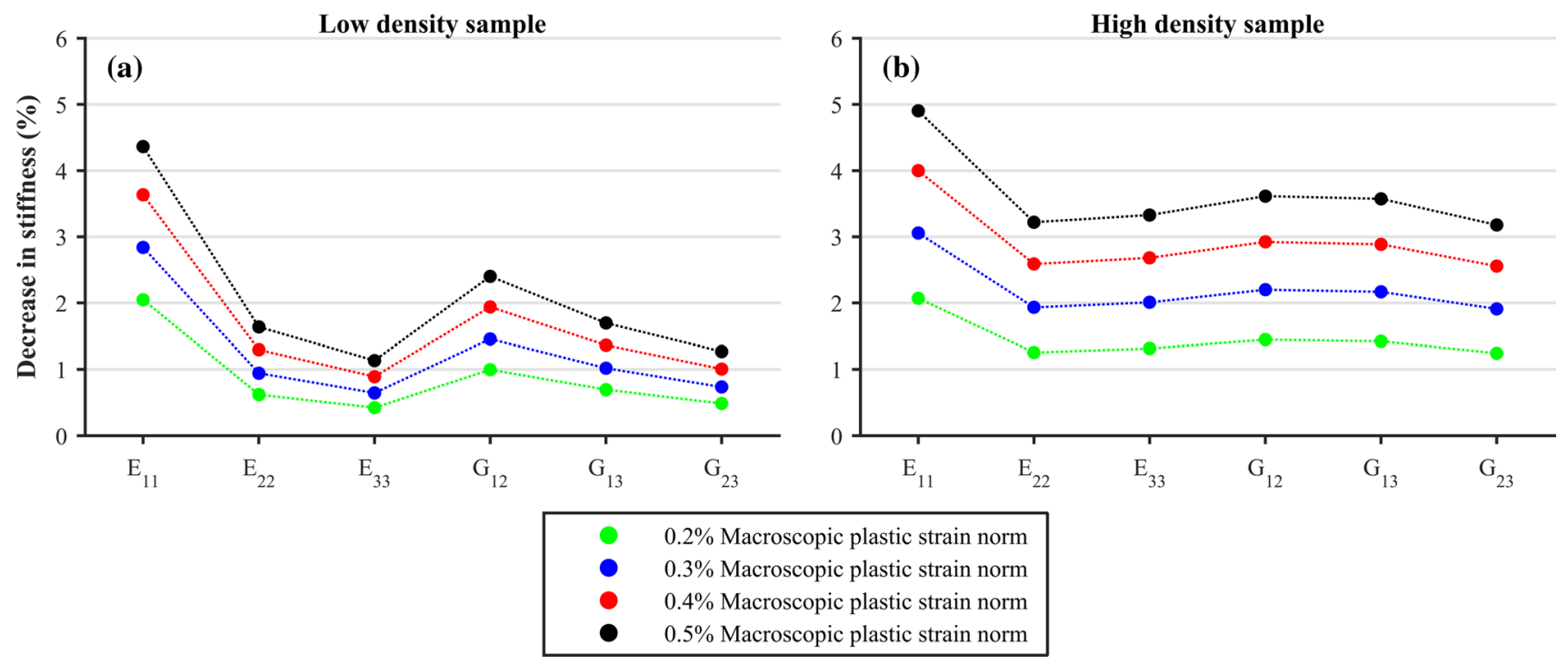

Fig. 4 Decrease in stiffness components for the most porous $(\mathrm{BV} / \mathrm{TV}=14.8 \%)$ and densest $(\mathrm{BV} / \mathrm{TV}=30.3 \%)$ samples due to tensile loading in direction 1 (i.e. $+\varepsilon_{11}$ ), and for all the considered macroscopic strain norm levels

\section{Discussion}

Damage behaviour of trabecular bone at the macroscale has been assessed in some previous studies for relatively simple load scenarios (Zioupos et al. 2008; Garcia et al. 2009; Sun et al. 2010). Damage at the microscopic level has also been previously considered (Gupta et al. 2006). This study aims to bridge both scales by investigating the damage behaviour of trabecular bone at the macroscale through a homogenisation-based multiscale approach and the use of multiple load cases for trabecular bone samples with very detailed geometry. Twelve $\mu$ FE meshes of samples covering a wide range of BV/TV and nine straincontrolled uniaxial load cases per sample were investigated with plasticity and damage included at the solid phase level.

The constitutive behaviour of the solid phase, including its damage behaviour, was considered to be isotropic. Cowin (1997) stated that the assumption of isotropy leads to little to no error. This is because trabeculae are composed of laminated material about their axes, which implies transverse isotropy or orthotropy; since the axis of a trabecula is the same as its loading axis, a beam made of orthotropic material can be reduced to a beam made of isotropic material. However, isotropic damage at the microscale results in anisotropic macroscale damage response, and depending on the loading scenario (Fig. 2). It is also interesting to note that strain-controlled uniaxial compressive or tensile loading results in damage not only in the direction of loading but also in other normal and shear directions. Shi et al. (2010) suggested that tissue yielding and microdamage only have a moderately strong correlation, whereas our tissue constitutive model directly links damage and yielding, as damage explicitly depends on the accumulated plastic strain. Nonetheless, they also suggested that there is a larger proportion of damaged tissue in the longitudinal trabeculae (direction of loading), which is in agreement with our results as the most damaged orthotropic stiffness component is always the on-axis component. Some previous studies which have modelled damage at the macroscale have assumed an isotropic behaviour (Schwiedrzik and Zysset 2013; Garcia et al. 2009), which may be an acceptable assumption for proportional loading, but not for changing loads, as would be expected during physiological activities.

Damage (Fig. 3) can be linearly related to the macroscopic strain norm with high coefficients of determination $\left(R^{2}>0.84\right)$, except for shear cases $\left(R^{2}<0.60\right)$. This also suggests that the evolution of damage at the macroscale can be assumed to be linear in the range of considered macroscopic strain norms $(0.2-0.5 \%)$. Beyond these strain levels, other effects, such as cracking and fracture of trabecula, can lead to structural failure and softening if further loading is applied (Kopperdahl and Keaveny 1998; Hosseini et al. 2014); this was not considered in this study since it is expected that the relatively low levels of macroscopic strain will not trigger these effects.

The values of the slopes of the linear fits are shown in Table 1; they show that damage propagation increases differently for strain-controlled uniaxial tension and compression load cases, with the value for compression cases being around twice the value for tension cases. This may be due to the fact that under compression, heterogeneous stress distributions occur that include tensile stresses at the solid phase level due to bending and buckling of trabeculae (Stölken and Kinney 

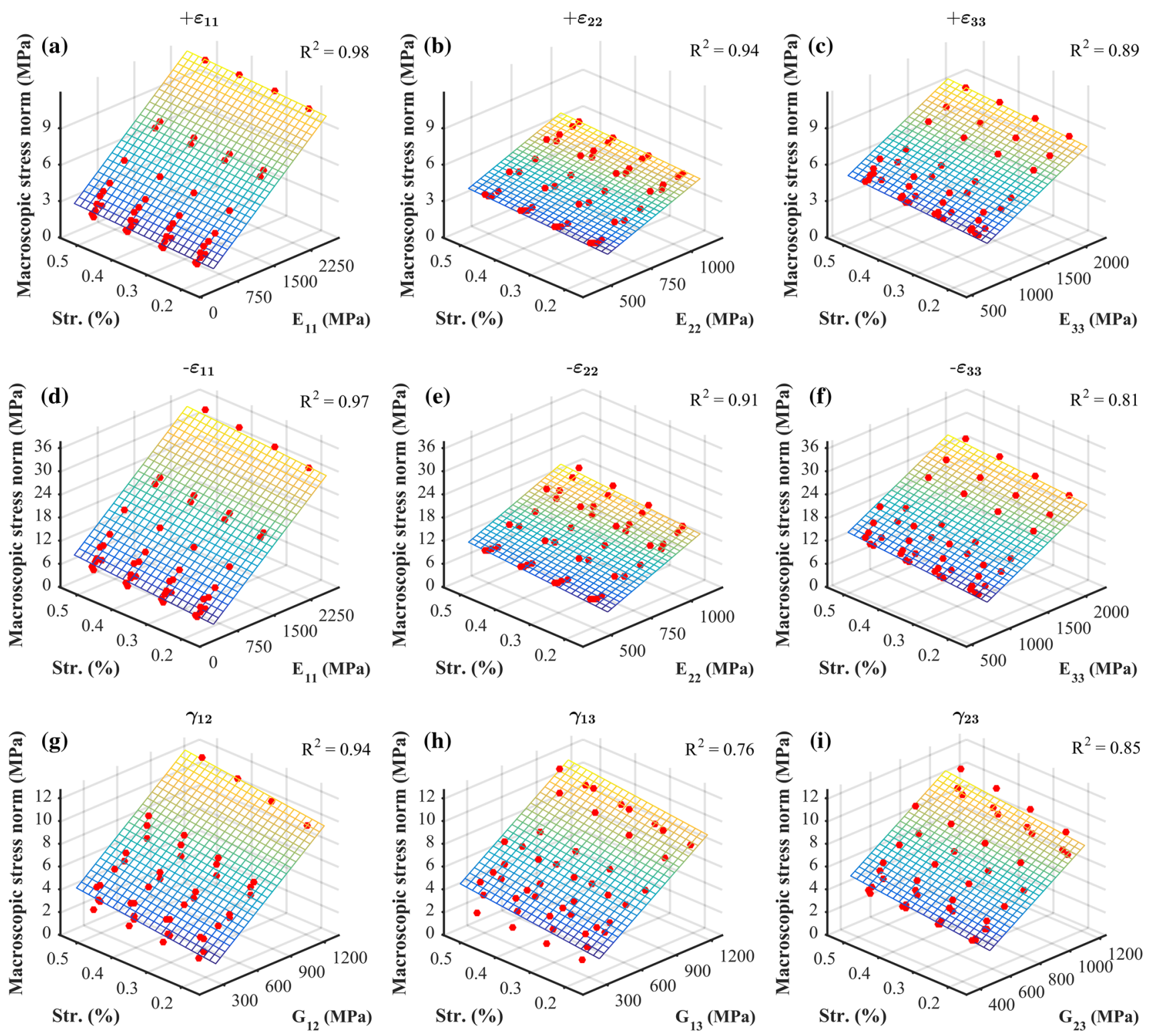

Fig. 5 Macroscopic yield stress norms for each of the considered load cases, for all the considered samples and for all the considered macroscopic strain norms (Str. stands for macroscopic strain norm). The considered uniaxial strain-controlled load cases are: tensile loading in

direction $1(\mathbf{a}), 2(\mathbf{b})$ and $3(\mathbf{c})\left(+\varepsilon_{11},+\varepsilon_{22}\right.$ and $+\varepsilon_{33}$, respectively); compressive loading in direction $1(\mathbf{d}), 2(\mathbf{e})$ and $3(\mathbf{f})\left(-\varepsilon_{11},-\varepsilon_{22}\right.$ and $-\varepsilon_{33}$, respectively); and shear loading in plane $1-2(\mathbf{g}), 1-3(\mathbf{h})$ and $2-3(\mathbf{i})\left(\gamma_{12}\right.$, $\gamma_{13}$ and $\gamma_{23}$, respectively)

Table 2 Values of the coefficient of determination $R^{2}$ and slopes for the linear fits between macroscopic yield stress norms, corresponding initial stiffness and macroscopic strain norms, for each of the considered load cases

\begin{tabular}{llllllllll}
\hline Load case & $+\epsilon_{11}$ & $+\epsilon_{22}$ & $+\epsilon_{33}$ & $-\epsilon_{11}$ & $-\epsilon_{22}$ & $-\epsilon_{33}$ & $\gamma_{12}$ & $\gamma_{13}$ & $\gamma_{23}$ \\
$R^{2}$ & 0.98 & 0.94 & 0.90 & 0.97 & 0.91 & 0.81 & 0.94 & 0.77 & 0.85 \\
Slope with respect to initial stiffness & $3.73 \mathrm{E}-3$ & $5.19 \mathrm{E}-3$ & $3.40 \mathrm{E}-3$ & $11.31 \mathrm{E}-3$ & $17.44 \mathrm{E}-3$ & $10.83 \mathrm{E}-3$ & $8.29 \mathrm{E}-3$ & $7.34 \mathrm{E}-3$ & $8.94 \mathrm{E}-3$ \\
$\begin{array}{l}\text { Slope with respect to macroscopic } \\
\text { strain norm (MPa) }\end{array}$ & 121.85 & 135.48 & 175.69 & 648.97 & 679.47 & 687.42 & 362.56 & 344.11 & 339.19 \\
\hline
\end{tabular}

2003; Bevill et al. 2006). Additionally, damage and plasticity in compression are far more diffused than in tension where they are more localised (Lambers et al. 2014). These lead to larger volumes of bone yielding (and thus being damaged as well) throughout the compression process when compared to tension. This is captured by the homogenisation procedure 

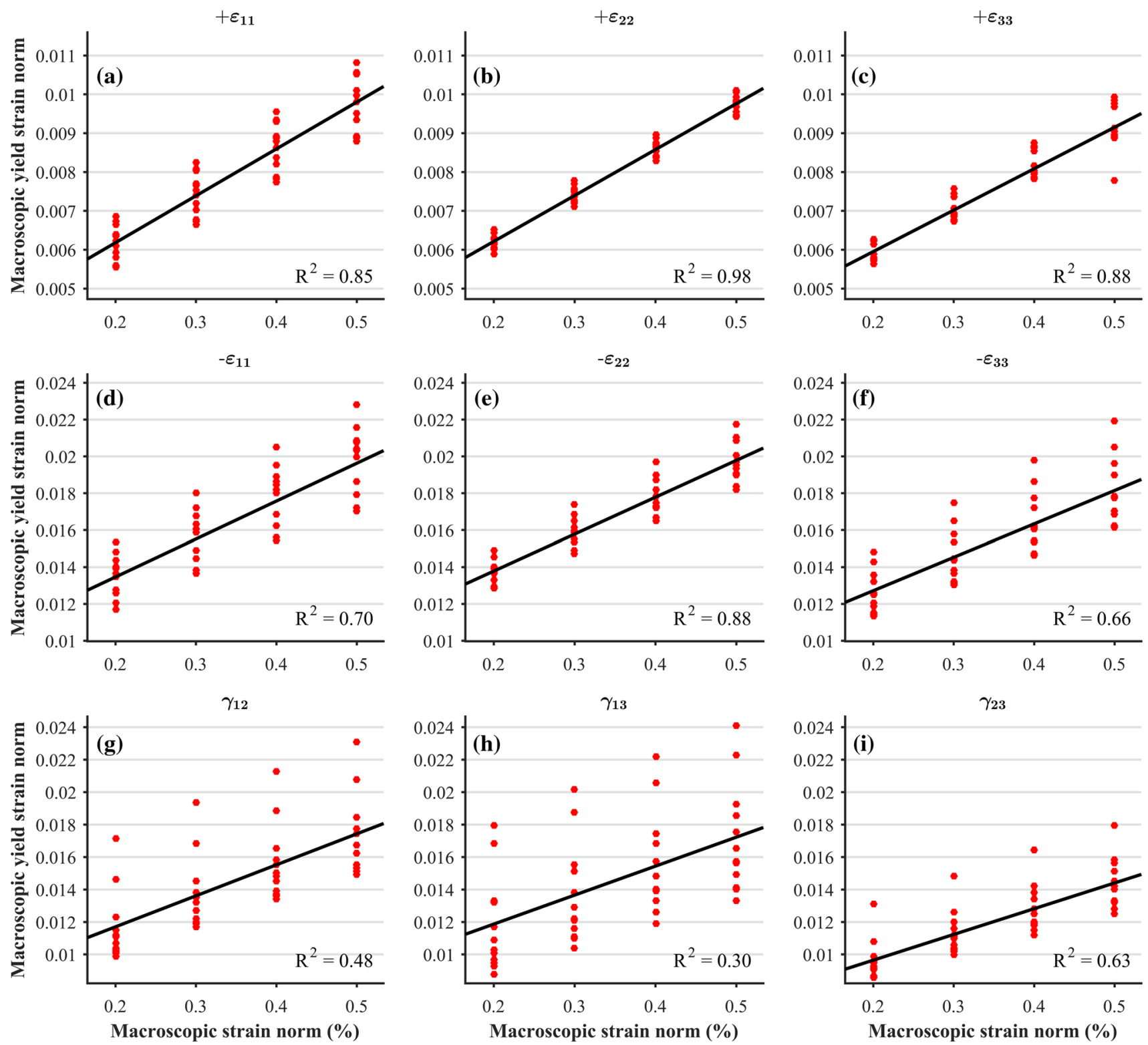

Fig. 6 Macroscopic yield strains for each of the considered load cases, for all the considered samples and for all the considered macroscopic strain norms. The considered uniaxial strain-controlled load cases are: respectively); compressive loading in direction $1(\mathbf{d}), 2(\mathbf{e})$ and $3(\mathbf{f})$ $\left(-\varepsilon_{11},-\varepsilon_{22}\right.$ and $-\varepsilon_{33}$, respectively); and shear loading in plane $1-2(\mathbf{g})$, $1-3(\mathbf{h})$ and $2-3(\mathbf{i})\left(\gamma_{12}, \gamma_{13}\right.$ and $\gamma_{23}$, respectively) tensile loading in direction $1(\mathbf{a}), 2(\mathbf{b})$ and $3(\mathbf{c})\left(+\varepsilon_{11},+\varepsilon_{22}\right.$ and $+\varepsilon_{33}$,

Table 3 Values of the coefficient of determination $R^{2}$ and slopes for the linear fits between macroscopic yield strain norms and macroscopic strain norms, for each of the considered load cases

\begin{tabular}{|c|c|c|c|c|c|c|c|c|c|}
\hline Load case & $+\epsilon_{11}$ & $+\epsilon_{22}$ & $+\epsilon_{33}$ & $-\epsilon_{11}$ & $-\epsilon_{22}$ & $-\epsilon_{33}$ & $\gamma_{12}$ & $\gamma_{13}$ & $\gamma_{23}$ \\
\hline$R^{2}$ & 0.85 & 0.98 & 0.88 & 0.70 & 0.88 & 0.65 & 0.48 & 0.30 & 0.63 \\
\hline $\begin{array}{l}\text { Slope with respect to macroscopic } \\
\text { strain norm }\end{array}$ & 1.21 & 1.18 & 1.07 & 2.06 & 2.00 & 1.81 & 1.91 & 1.79 & 1.59 \\
\hline
\end{tabular}

and expressed as a higher slope for the damage progression. In tension, cracks are more localised and propagate faster, eventually leading to catastrophic failure of individual trabec- ulae. Moreover, it is also likely that cracks under compression exhibit some partial closure since bone is a quasi-brittle material, leading to reduced effects of damage on the stiffness. 
Nonetheless, these effects have not been included in the solid phase constitutive model.

If individual samples are considered (Fig. 4), it can be seen that damage evolves with increasing macroscopic strain norm and that the low BV/TV sample has a considerable difference in damage between the stiffness component corresponding to the load case the sample is subjected to and the rest of stiffness components, effect which is not observed in the high $\mathrm{BV} / \mathrm{TV}$ sample. However, the expectation that high BV/TV, more continuum-like, trabecular bone samples would partially upscale the isotropic damage behaviour of the solid phase was not supported by the statistical analysis as BV/TV was not found to be a good predictor of this. Perhaps, more samples with a wider range of BV/TV and rod/plate morphology could lead to morphological parameters being related to damage.

Several previous studies (Wolfram et al. 2012; LevreroFlorencio et al. 2016; Panyasantisuk et al. 2015) used the $0.2 \%$ criterion to determine the macroscopic yield of trabecular bone. However, this study shows that if damage is included, it results in a certain reduction of stiffness at the $0.2 \%$ macroscopic strain norm (Table 1 ). This implies that a modified elasticity tensor may need to be used once macroscopic yield is encountered. This can be done by considering a damaged slope obtained by joining the origin and the yield stress at $0.2 \%$ macroscopic strain norm. Previous studies have employed an isotropic reduction of the elastic stiffness (Wolfram et al. 2012); however, this study shows that the macroscopic damage may not be isotropic.

With respect to the hardening of trabecular bone at the macroscale, the fits show that yield points described in both stress and strain spaces show linear hardening for this range of macroscopic strain norms. However, as for damage propagation, the slopes are found to be different for different load cases (Table 2); hardening in compression, tension and shear are considerably different. Hardening in compression is considerably larger than the rest, which is likely to be due to the fact that Drucker-Prager is used as the solid phase yield criterion, implying that the lack of hydrostatic compression yield may be partially upscaled to the macroscale, resulting in an increased evolution of the stress norm throughout the loading process. Although most models of trabecular bone at the macroscale use isotropic hardening (Garcia et al. 2009; Schwiedrzik et al. 2013) and nonlinear hardening laws (Garcia et al. 2009; Schwiedrzik and Zysset 2013), our results show a hardening behaviour which depends on the considered load case (i.e. tension, compression or shear) and a linear relationship between macroscopic yield stress/yield strain norm and macroscopic strain norm. However, the considered range of post-elastic strains is small in this study and hardening may become nonlinear if further loading is applied.

The validation of the results in this study is especially difficult to carry out. Damage evolution at the macroscale is usually evaluated by using cyclic loading experiments (Keaveny et al. 1994; Zysset and Curnier 1996). The configuration of these experiments is very different to the ones used in $\mu$ FE-related studies due to the BCs of the specimens. A similar problem occurs when validating the hardening results. Additionally, hardening in the literature is usually reported in the form of stress-strain curves, which indeed depend on the morphology of the specimens (high BV/TV specimens are likely to yield at larger stresses). Thus, samples of similar morphologies would be required to establish a meaningful comparison. Moreover, hardening values are usually reported for a larger range of strains, which are way beyond our small range of post-elastic strains. Nonetheless, we foresee that, in general, our hardening values are larger as they correspond to the inclined portion of the stress-strain curve immediately after yield.

The orthotropic assumption for the macroscopic elastic stiffness was used. The macroscopic strain is readily available, since it is directly applied through the considered BC (Wang et al. 2009). Nonetheless, in some shear load cases the homogenised stress presents some nonzero normal components (larger than one order of magnitude below the shear components of the stress tensor). This implies that even for these highly aligned samples, in-plane trabeculae experience normal stresses under macroscopic shear. It was shown by Sanyal et al. (2012) that macroscopic shear load cases are dominated by tensile solid phase stresses in trabeculae which are aligned $45^{\circ}$ from the shear in-plane axes. The homogenisation procedure is likely to have captured these tensile stresses when assessing the homogenised stress of the considered VE. This suggests that, at the macroscopic level, the normal and shear behaviours do have some interaction, which may outline a possible limit of the orthotropic macroscopic elastic assumption for trabecular bone.

Our study has a number of limitations. We use bovine trabecular bone specimens, whose results may not be readily comparable to human bone. Damage behaviour of bone at the tissue level has been researched in some previous studies (Schwiedrzik and Zysset 2013, 2015), but the maximum damage value is not known (Schwiedrzik and Zysset 2015) so we treated it as $90 \%$ reduction of the initial stiffness as an approximation and to avoid numerical difficulties related to the complete loss of stress carrying capacity; more research on the damage behaviour at the solid phase level is needed. We checked the effect of different thresholds (including $100 \%$ reduction) for one sample and found that the nonisotropic and loading mode dependency trend in damage is maintained and the difference between damage values at different macroscopic strain norms is small. There is plenty of experimental data on uniaxial load cases in literature (Keaveny et al. 1997; Bayraktar and Keaveny 2004; Sanyal et al. 2012; Manda et al. 2016), but these experiments do not permit the evaluation of the damaged orthotropic stiffness tensor. 
Additionally, these experiments do not allow for evaluation of samples submitted to different load cases, as yield or damage in one direction may affect the behaviour in other directions. Therefore, it may be argued that some of the results in this study are of higher order. Another limitation is the use of kinematic uniform $\mathrm{BCs}$ in the homogenisation procedure, which are known for being too stiff (Panyasantisuk et al. 2015), and may affect the damage morphology when compared to the relevant in situ case (Daszkiewicz et al. 2016). As in previous studies (Wolfram et al. 2012; Levrero-Florencio et al. 2016; Panyasantisuk et al. 2015), this study assumes the solid phase to be homogeneous. It has been shown that trabecular bone tissue has heterogeneous mineral density, and thus heterogeneous properties (Blanchard et al. 2013; Renders et al. 2008). However, the effects of mineral heterogeneities have minor influence on the apparent elastic properties of trabecular bone (Gross et al. 2012). Additionally, the effects of these heterogeneities in models with geometrical or material nonlinearities are still unknown and further research is needed to establish comparisons. The solid phase was modelled as a damage-plastic material without fracture, which is perhaps appropriate for the level of strains applied. It has been previously shown that ductile solid phase behaviour overestimates the experimental yield properties, especially at low BV/TV (Nawathe et al. 2013). Only strain-controlled uniaxial load cases for a relatively small number of samples were evaluated. In order to describe the full multiaxial behaviour of trabecular bone, more load cases are needed. However, the computational cost of performing a complete nonlinear simulation with the high resolution used in this study and with damage, plasticity, and an elastic homogenisation at each time increment is very high (it is important to point out that as damage grows, the stiffness matrix becomes increasingly unsymmetric, which decreases the convergence rate of the used iterative linear algebraic solver).

Acknowledgements The authors gratefully acknowledge the EPSRC for the Grant EP/K036939/1 and for access to ARCHER under the project "Modelling the nonlinear micromechanical behaviour of bone". The first author was funded through a Principal's Career Development Scholarship by The University of Edinburgh.

\section{Compliance with ethical standards}

Conflict of interest The authors declare that there is no conflict of interest.

Open Access This article is distributed under the terms of the Creative Commons Attribution 4.0 International License (http://creativecomm ons.org/licenses/by/4.0/), which permits unrestricted use, distribution, and reproduction in any medium, provided you give appropriate credit to the original author(s) and the source, provide a link to the Creative Commons license, and indicate if changes were made.

\section{Appendix: Implementation of the solid phase con- stitutive law}

The mathematical operators are found in Section 2.2. The matrix representation of higher-order tensors is denoted with a bold upper-case character within square brackets, such as [A], and the vector representation of second-order tensors is denoted with a bold lower-case character within curly brackets, such as $\{\mathbf{a}\}$. However, note that [ ] can also represent, in conjunction with ( ), priority in the order of mathematical operations.

\section{Definition of the model}

The constitutive model in this "Appendix" is based on the work of Schwiedrzik and Zysset (2013). In this study, we use a yield surface based on Schwiedrzik et al. (2013) with a damage cap based on Schwiedrzik and Zysset (2015).

The yield function is

$f(\boldsymbol{\sigma})=\sqrt{\boldsymbol{\sigma}: \mathbb{F}: \boldsymbol{\sigma}}+\mathbf{F}: \boldsymbol{\sigma}-\left(1+H \bar{\varepsilon}^{p}\right)=0$,

where $\sigma$ is the Kirchhoff stress, and $H$ is the hardening modulus (which is constant due to the assumption of linear isotropic hardening), and $D$ is the damage variable, defined as

$D=D_{c}\left(1-\mathrm{e}^{-k_{p} \bar{\varepsilon}^{p}}\right)$,

where $D_{c}$ and $k_{p}$ are positive constants related to damage evolution.

\section{Definition of the evolution equations}

The residuals of the damaged quadric yield surface can be obtained by using an implicit time integration for the evolution equations. The closest-point projection method (CPPM) equations are defined as (note that the time increment subscripts are omitted for convenience)

$$
\left\{\begin{array}{c}
\mathbf{R}_{\boldsymbol{\sigma}} \\
f
\end{array}\right\}=\left\{\begin{array}{c}
\sigma-(1-D) \mathbb{D}^{e}:\left(\boldsymbol{\varepsilon}^{e \text { trial }}-\Delta \bar{\varepsilon}^{p} \frac{\mathbf{N}}{\|\mathbf{N}\|}\right) \\
\sqrt{\boldsymbol{\sigma}: \mathbb{F}: \boldsymbol{\sigma}}+\mathbf{F}: \boldsymbol{\sigma}-\left(1+H \bar{\varepsilon}^{p}\right)
\end{array}\right\}
$$

where $\mathbf{R}_{\sigma}$ is the residual of stresses, $\boldsymbol{\varepsilon}^{e \text { trial }}$ is the elastic trial strain, $\Delta \bar{\varepsilon} p$ is the increment of accumulated plastic strain, and $\mathbf{N}=\frac{\partial f}{\partial \boldsymbol{\sigma}}=\frac{\mathbb{F}: \boldsymbol{\sigma}}{\sqrt{\boldsymbol{\sigma}: \mathbb{F}: \boldsymbol{\sigma}}}+\mathbf{F}$. The residual of stresses can be converted to residual of elastic strains by using the term $(1-D)^{-1}\left(\mathbb{D}^{e}\right)^{-1}$, so that

$$
\left\{\begin{array}{c}
\mathbf{R}_{\boldsymbol{\varepsilon}^{e}} \\
f
\end{array}\right\}=\left\{\begin{array}{c}
\boldsymbol{\varepsilon}^{e}-\boldsymbol{\varepsilon}^{e \text { trial }}+\Delta \bar{\varepsilon}^{p} \frac{\mathbf{N}}{\|\mathbf{N}\|} \\
\sqrt{\boldsymbol{\sigma}: \mathbb{F}: \boldsymbol{\sigma}}+\mathbf{F}: \sigma-\left[1+H\left(\bar{\varepsilon}^{p \text { trial }}+\Delta \bar{\varepsilon}^{p}\right)\right]
\end{array}\right\}
$$


where $\bar{\varepsilon}^{p \text { trial }}$ is the trial value of $\bar{\varepsilon}^{p}$. The solution variables are defined as

$\mathbf{x}=\left\{\begin{array}{c}\varepsilon^{e} \\ \Delta \bar{\varepsilon}^{p}\end{array}\right\}$

The residuals are linearised as

$$
\begin{aligned}
& \left\{\begin{array}{c}
\mathrm{d} \boldsymbol{\varepsilon}^{e \text { trial }} \\
0
\end{array}\right\} \\
& =\left\{\begin{array}{c}
{\left[\mathbb{I}+\Delta \bar{\varepsilon}^{p} \frac{\partial}{\partial \boldsymbol{\varepsilon}^{e}}\left(\frac{\mathbf{N}}{\|\mathbf{N}\|}\right)\right]: \mathrm{d} \boldsymbol{\varepsilon}^{e}+\mathrm{d} \Delta \bar{\varepsilon}^{p}\left[\frac{\mathbf{N}}{\|\mathbf{N}\|}+\Delta \bar{\varepsilon}^{p} \frac{\partial}{\partial \Delta \bar{\varepsilon}^{p}}\left(\frac{\mathbf{N}}{\|\mathbf{N}\|}\right)\right]} \\
\mathbf{N}:\left(\frac{\partial \boldsymbol{\sigma}}{\partial \boldsymbol{\varepsilon}^{e}}: \mathrm{d} \boldsymbol{\varepsilon}^{e}+\frac{\partial \sigma}{\partial D} D^{\prime} \mathrm{d} \Delta \bar{\varepsilon}^{p}\right)-H \mathrm{~d} \Delta \bar{\varepsilon}^{p}
\end{array}\right\},
\end{aligned}
$$

where $\mathbb{I}_{i j k l}=\delta_{i k} \delta_{j l}$ and $\delta$ is the Kronecker delta. The increment in plastic strain, and the corresponding accumulated plastic strain, are defined as

$\Delta \boldsymbol{\varepsilon}^{p}=\Delta \bar{\varepsilon}^{p} \frac{\mathbf{N}}{\|\mathbf{N}\|}$

and

$\bar{\varepsilon}^{p}=\bar{\varepsilon}^{p \text { trial }}+\Delta \gamma\|\mathbf{N}\|$.

After rearranging these terms, Eq. 15 becomes

$$
\left\{\begin{array}{c}
\mathrm{d} \boldsymbol{\varepsilon}^{e \text { trial }} \\
0
\end{array}\right\}=\left[\mathbf{J}_{C P P M}\right]\left\{\begin{array}{c}
\mathrm{d} \boldsymbol{\varepsilon}^{e} \\
\mathrm{~d} \Delta \bar{\varepsilon}^{p}
\end{array}\right\},
$$

where $\left[\mathbf{J}_{C P}\right.$ P $\left.M\right]$ is the Jacobian of the CPPM scheme, defined as

$\left[\mathbf{J}_{C P P M}\right]=\left[\begin{array}{cc}\mathbb{I}+\Delta \bar{\varepsilon}^{p} \frac{\partial}{\partial \boldsymbol{\varepsilon}^{e}}\left(\frac{\mathbf{N}}{\|\mathbf{N}\|}\right) \frac{\mathbf{N}}{\|\mathbf{N}\|}+\frac{\partial}{\partial \Delta \bar{\varepsilon}^{p}}\left(\frac{\mathbf{N}}{\|\mathbf{N}\|}\right) \\ (1-D) \mathbf{N}: \mathbb{D}^{e} & \mathbf{N}: \frac{\partial \sigma}{\partial D} D^{\prime}-H\end{array}\right]$.

The derivatives employed in the previous equation are

$$
\begin{aligned}
& \frac{\partial}{\partial \boldsymbol{\varepsilon}^{e}}\left(\frac{\mathbf{N}}{\|\mathbf{N}\|}\right)=\frac{1-D}{\|\mathbf{N}\|}\left(\mathbb{I}-\frac{1}{(\|\mathbf{N}\|)^{2}} \mathbf{N} \otimes \mathbf{N}\right): \frac{\partial \mathbf{N}}{\partial \boldsymbol{\sigma}}: \mathbb{D}^{e}, \\
& \frac{\partial \mathbf{N}}{\partial \boldsymbol{\sigma}}=\frac{\mathbb{F}}{\sqrt{\sigma: \mathbb{F}: \boldsymbol{\sigma}}}-\frac{(\mathbb{F}: \boldsymbol{\sigma}) \otimes(\boldsymbol{\sigma}: \mathbb{F})}{(\sqrt{\boldsymbol{\sigma}: \mathbb{F}: \boldsymbol{\sigma}})^{3}}, \\
& \frac{\partial}{\partial \Delta \bar{\varepsilon}^{p}}\left(\frac{\mathbf{N}}{\|\mathbf{N}\|}\right)=\mathbf{0}, \\
& \frac{\partial \boldsymbol{\sigma}}{\partial D}=-\mathbb{D}^{e}: \boldsymbol{\varepsilon}^{e},
\end{aligned}
$$

and

$D^{\prime}=\frac{\partial D}{\partial \Delta \bar{\varepsilon}^{p}}=D_{c} k_{p} \mathrm{e}^{-k_{p}\left(\bar{\varepsilon}^{p \text { trial }}+\Delta \bar{\varepsilon}^{p}\right)}$.

\section{Consistent tangent operator}

From the first row of Eq. 18, we obtain

$$
\begin{aligned}
& {\left[\mathbb{I}+\Delta \bar{\varepsilon}^{p} \frac{1-D}{\|\mathbf{N}\|}\left(\mathbb{I}-\frac{1}{(\|\mathbf{N}\|)^{2}} \mathbf{N} \otimes \mathbf{N}\right): \frac{\partial \mathbf{N}}{\partial \boldsymbol{\sigma}}: \mathbb{D}^{e}\right]: \mathrm{d} \boldsymbol{\varepsilon}^{e}} \\
& +\frac{\mathbf{N}}{\|\mathbf{N}\|} \mathrm{d} \Delta \bar{\varepsilon}^{p}=\mathrm{d} \boldsymbol{\varepsilon}^{e \text { trial }}
\end{aligned}
$$

The first term of the LHS can be further developed by using

$\mathrm{d} \boldsymbol{\varepsilon}^{e}=\frac{1}{1-D}\left(\mathbb{D}^{e}\right)^{-1}:\left(\mathrm{d} \boldsymbol{\sigma}-\frac{\partial \boldsymbol{\sigma}}{\partial \Delta \bar{\varepsilon}^{p}} \mathrm{~d} \Delta \bar{\varepsilon}^{p}\right)$,

such as

$$
\begin{aligned}
{[} & \left.\mathbb{I}+\Delta \bar{\varepsilon}^{p} \frac{1-D}{\|\mathbf{N}\|}\left(\mathbb{I}-\frac{1}{(\|\mathbf{N}\|)^{2}} \mathbf{N} \otimes \mathbf{N}\right): \frac{\partial \mathbf{N}}{\partial \boldsymbol{\sigma}}: \mathbb{D}^{e}\right]: \mathrm{d} \boldsymbol{\varepsilon}^{e} \\
= & {\left[\frac{1}{1-D}\left(\mathbb{D}^{e}\right)^{-1}+\Delta \bar{\varepsilon}^{p} \frac{1}{\|\mathbf{N}\|}\left(\mathbb{I}-\frac{1}{(\|\mathbf{N}\|)^{2}} \mathbf{N} \otimes \mathbf{N}\right): \frac{\partial \mathbf{N}}{\partial \boldsymbol{\sigma}}\right]: } \\
& \left(\mathrm{d} \boldsymbol{\sigma}-\frac{\partial \boldsymbol{\sigma}}{\partial \Delta \bar{\varepsilon}^{p}} \mathrm{~d} \Delta \bar{\varepsilon}^{p}\right),
\end{aligned}
$$

which, when applied to Eq. 22, becomes

$$
\begin{aligned}
\mathrm{d} \boldsymbol{\sigma} & =\mathbb{P}:\left(\mathrm{d} \boldsymbol{\varepsilon}^{e \text { trial }}-\mathrm{d} \Delta \bar{\varepsilon}^{p} \frac{\mathbf{N}}{\|\mathbf{N}\|}\right)+\frac{\partial \boldsymbol{\sigma}}{\partial \Delta \bar{\varepsilon}^{p}} \mathrm{~d} \Delta \bar{\varepsilon}^{p} \\
& =\mathbb{P}: \mathrm{d} \boldsymbol{\varepsilon}^{e \text { trial }}+\left(\frac{\partial \boldsymbol{\sigma}}{\partial \Delta \bar{\varepsilon}^{p}}-\mathbb{P}: \frac{\mathbf{N}}{\|\mathbf{N}\|}\right) \mathrm{d} \Delta \bar{\varepsilon}^{p}
\end{aligned}
$$

where the fourth-order tensor $\mathbb{P}$ is defined as

$\mathbb{P}=\left[\frac{1}{1-D}\left(\mathbb{D}^{e}\right)^{-1}+\Delta \bar{\varepsilon}^{p} \frac{1}{\|\mathbf{N}\|}\left(\mathbb{I}-\frac{1}{(\|\mathbf{N}\|)^{2}} \mathbf{N} \otimes \mathbf{N}\right): \frac{\partial \mathbf{N}}{\partial \boldsymbol{\sigma}}\right]^{-1}$

From the second row of Eq. 18, we obtain

$\mathbf{N}: \mathrm{d} \boldsymbol{\sigma}+\left(\mathbf{N}: \frac{\partial \boldsymbol{\sigma}}{\partial D} D^{\prime}-H\right) \mathrm{d} \Delta \bar{\varepsilon}^{p}=0$.

By using Eq. 25 and Eq. 27, we obtain

$$
\begin{gathered}
\mathbf{N}:\left[\mathbb{P}: \mathrm{d} \boldsymbol{\varepsilon}^{e \text { trial }}+\mathrm{d} \Delta \bar{\varepsilon}^{p}\left(-\mathbb{P}: \frac{\mathbf{N}}{\|\mathbf{N}\|}+\frac{\partial \boldsymbol{\sigma}}{\partial \Delta \bar{\varepsilon}^{p}}\right)\right] \\
+\left(\mathbf{N}: \frac{\partial \boldsymbol{\sigma}}{\partial \Delta \bar{\varepsilon}^{p}}-H\right) \mathrm{d} \Delta \bar{\varepsilon}^{p}=0 \\
\rightarrow \mathrm{d} \Delta \bar{\varepsilon}^{p}=\frac{\mathbf{N}: \mathbb{P}: \mathrm{d} \boldsymbol{\varepsilon}^{e \text { trial }}}{\mathbf{N}:\left(\mathbb{P}: \frac{\mathbf{N}}{\|\mathbf{N}\|}-2 \frac{\partial \boldsymbol{\sigma}}{\partial \Delta \bar{\varepsilon}^{p}}\right)+H} .
\end{gathered}
$$


If this expression is inserted into Eq. 25, then

$$
\mathrm{d} \boldsymbol{\sigma}=\mathbb{P}: \mathrm{d} \boldsymbol{\varepsilon}^{e \text { trial }}+\frac{\mathbf{N}: \mathbb{P}: \mathrm{d} \boldsymbol{\varepsilon}^{e \text { trial }}}{\mathbf{N}:\left(\mathbb{P}: \frac{\mathbf{N}}{\|\mathbf{N}\|}-2 \frac{\partial \sigma}{\partial \Delta \bar{\varepsilon}^{p}}\right)+H}\left(\frac{\partial \boldsymbol{\sigma}}{\partial \Delta \bar{\varepsilon}^{p}}-\mathbb{P}: \frac{\mathbf{N}}{\|\mathbf{N}\|}\right) .
$$

The final expression of the (generally unsymmetric) consistent elastoplastic tangent tensor, $\mathbb{D}^{e p}$, is obtained by taking into account that $\mathbb{D}^{e p}=\frac{\partial \sigma}{\partial \varepsilon^{e} \text { trial }}$, such that

$$
\mathbb{D}^{e p}=\mathbb{P}+\frac{\left(\frac{\partial \sigma}{\partial \Delta \bar{\varepsilon}^{p}}-\mathbb{P}: \frac{\mathbf{N}}{\|\mathbf{N}\|}\right) \otimes(\mathbf{N}: \mathbb{P})}{\mathbf{N}:\left(\mathbb{P}: \frac{\mathbf{N}}{\|\mathbf{N}\|}-2 \frac{\partial \sigma}{\partial \Delta \bar{\varepsilon}^{p}}\right)+H}
$$

\section{References}

Bayraktar HH, Keaveny TM (2004) Mechanisms of uniformity of yield strains for trabecular bone. J Biomech 37(11):1671-1678

Bevill G, Eswaran SK, Gupta A, Papadopoulos P, Keaveny TM (2006) Influence of bone volume fraction and architecture on computed large-deformation failure mechanisms in human trabecular bone. Bone 39(6): 1218-1225

Blanchard R, Dejaco A, Bongaers E, Hellmich C (2013) Intravoxel bone micromechanics for microCT-based finite element simulations. J Biomech 46(15):2710-2721

Carnelli D, Gastaldi D, Sassi V, Contro R, Ortiz C, Vena P (2010) A finite element model for direction-dependent mechanical response to nanoindentation of cortical bone allowing for anisotropic postyield behavior of the tissue. J Biomech Eng. doi:10.1115/1. 4001358

Completo A, Simões JA, Fonseca F (2009) Revision total knee arthroplasty: the influence of femoral stems in load sharing and stability. Knee 16(4):275-279

Conlisk N, Howie CR, Pankaj P (2015) The role of complex clinical scenarios in the failure of modular components following revision total knee arthroplasty: a finite element study. J Orthop Res 33(8):1134-1141

Cowin S (1985) The relationship between the elasticity tensor and the fabric tensor. Mech Mater 4(2):137-147

Cowin S (1986) Fabric dependence of an anisotropic strength criterion. Mech Mater 5(3):251-260

Cowin S (1997) Remarks on the paper entitled 'Fabric and elastic principal directions of cancellous bone are closely related'. J Biomech 30(11-12):1191-1192

Daszkiewicz K, Maquer G, Zysset PK (2016) The effective elastic properties of human trabecular bone may be approximated using micro-finite element analyses of embedded volume elements. Biomech Model Mechanobiol. doi:10.1007/ s10237-016-0849-3

de Souza Neto EA, Peric D, Owen DRJ (2008) Computational methods for plasticity: theory and applications, 1st edn. Wiley, Chichester

Doube M, Kłosowski MM, Arganda-Carreras I, Cordelières FP, Dougherty RP, Jackson JS, Schmid B, Hutchinson JR, Shefelbine SJ (2010) BoneJ: free and extensible bone image analysis in ImageJ. Bone 47(6):1076-1079

Garcia D, Zysset PK, Charlebois M, Curnier A (2009) A threedimensional elastic plastic damage constitutive law for bone tissue. Biomech Model Mechanobiol 8(2):149-165
Gómez W, Sales E, Lopes RT, Pereira WCA (2013) A comparative study of automatic thresholding approaches for 3D X-ray microtomography of trabecular bone. Med Phys. doi:10.1118/1.4817235

Gross T, Pahr DH, Peyrin F, Zysset PK (2012) Mineral heterogeneity has a minor influence on the apparent elastic properties of human cancellous bone: a SR $\mu$ CT-based finite element study. Comput Methods Biomech Biomed Eng 15(11):1137-1144

Gupta HS, Wagermaier W, Zickler GA, Hartmann J, Funari SS, Roschger P, Wagner HD, Fratzl P (2006) Fibrillar level fracture in bone beyond the yield point. Int J Fract 139(3-4):425-436

Harrigan TP, Mann RW (1984) Characterization of microstructural anisotropy in orthotropic materials using a second rank tensor. J Mater Sci 19(3):761-767

Harrigan TP, Jasty M, Mann RW, Harris WH (1988) Limitations of the continuum assumption in cancellous bone. J Biomech 21(4):269275

Helgason B, Perilli E, Schileo E, Taddei F, Brynjólfsson S, Viceconti M (2008) Mathematical relationships between bone density and mechanical properties: a literature review. Clin Biomech 23(2):135-146

Hollister SJ, Kikuchi N (1992) A comparison of homogenization and standard mechanics analyses for periodic porous composites. Comput Mech 10(2):73-95

Hollister SJ, Brennan JM, Kikuchi N (1994) A homogenization sampling procedure for calculating trabecular bone effective stiffness and tissue level stress. J Biomech 27(4):433-444

Hosseini HS, Clouthier AL, Zysset PK (2014) Experimental validation of finite element analysis of human vertebral collapse under large compressive strains. J Biomech Eng. doi:10.1115/1.4026409

Keaveny TM, Wachtel EF, Guo XE, Hayes WC (1994) Mechanical behavior of damaged trabecular bone. J Biomech 27(11):1309_ 1318

Keaveny TM, Pinilla TP, Crawford RP, Kopperdahl DL, Lou A (1997) Systematic and random errors in compression testing of trabecular bone. J Orthop Res 15(1):101-110

Keaveny TM, Wachtel EF, Kopperdahl DL (1999) Mechanical behavior of human trabecular bone after overloading. J Orthop Res 17(3):346-353

Keller TS (1994) Predicting the compressive mechanical behavior of bone. J Biomech 27(9):1159-1168

Kopperdahl DL, Keaveny TM (1998) Yield strain behavior of trabecular bone. J Biomech 31(7):601-608

Lambers FM, Bouman AR, Tkachenko EV, Keaveny TM, Hernandez CJ (2014) The effects of tensile-compressive loading mode and microarchitecture on microdamage in human vertebral cancellous bone. J Biomech 47(15):3605-3612

Levrero-Florencio F, Margetts L, Sales E, Xie S, Manda K, Pankaj P (2016) Evaluating the macroscopic yield behaviour of trabecular bone using a nonlinear homogenisation approach. J Mech Behav Biomed Mater 61:384-396

Maghous S, Dormieux L, Barthélémy J (2009) Micromechanical approach to the strength properties of frictional geomaterials. Eur J Mech A Solids 28(1):179-188

Manda K, Xie S, Wallace RJ, Levrero-Florencio F, Pankaj P (2016) Linear viscoelasticity - bone volume fraction relationships of bovine trabecular bone. Biomech Model Mechanobiol 15(6):1631-1640

Margetts L (2002) Parallel finite element analysis. Ph.D. thesis, University of Manchester. https://www.escholar.manchester.ac.uk/ uk-ac-man-scw:70784

Nawathe S, Juillard F, Keaveny TM (2013) Theoretical bounds for the influence of tissue-level ductility on the apparent-level strength of human trabecular bone. J Biomech 46(7):1293-1299

Odgaard A, Kabel J, van Rietbergen B, Dalstra M, Huiskes R (1997) Fabric and elastic principal directions of cancellous bone are closely related. J Biomech 30(5):487-495 
Pankaj P (2013) Patient-specific modelling of bone and bone-implant systems: the challenges. Int $\mathrm{J}$ Numer Methods Biomed Eng 29(1):233-249

Pankaj P, Donaldson FE (2013) Algorithms for a strain-based plasticity criterion for bone. Int J Numer Methods Biomed Eng 29(1):40-61

Panyasantisuk J, Pahr DH, Zysset PK (2015) Effect of boundary conditions on yield properties of human femoral trabecular bone. Biomech Model Mechanobiol 15(5):1043-1053

Pérez-Foguet A, Armero F (2002) On the formulation of closest-point projection algorithms in elastoplasticity—part II: globally convergent schemes. Int J Numer Methods Eng 53(2):331-374

Ponthot JP (1998) An extension of the radial return algorithm to account for rate-dependent effects in frictional contact and visco-plasticity. J Mater Process Technol 80-81:628-634

Reisinger AG, Pahr DH, Zysset PK (2010) Sensitivity analysis and parametric study of elastic properties of an unidirectional mineralized bone fibril-array using mean field methods. Biomech Model Mechanobiol 9(5):499-510

Renders GAP, Mulder L, Langenbach GEJ, van Ruijven LJ, van Eijden TMGJ (2008) Biomechanical effect of mineral heterogeneity in trabecular bone. J Biomech 41(13):2793-2798

Rincón-Kohli L, Zysset PK (2009) Multi-axial mechanical properties of human trabecular bone. Biomech Model Mechanobiol 8(3):195208

Sanyal A, Gupta A, Bayraktar HH, Kwon RY, Keaveny TM (2012) Shear strength behavior of human trabecular bone. J Biomech 45(15):2513-2519

Sanyal A, Scheffelin J, Keaveny TM (2015) The quartic piecewiselinear criterion for the multiaxial yield behavior of human trabecular bone. J Biomech Eng. doi:10.1115/1.4029109

Schileo E, Dall'ara E, Taddei F, Malandrino A, Schotkamp T, Baleani M, Viceconti M (2008) An accurate estimation of bone density improves the accuracy of subject-specific finite element models. J Biomech 41(11):2483-2491

Schwiedrzik JJ, Zysset PK (2013) An anisotropic elastic-viscoplastic damage model for bone tissue. Biomech Model Mechanobiol 12(2):201-213

Schwiedrzik JJ, Zysset PK (2015) The influence of yield surface shape and damage in the depth-dependent response of bone tissue to nanoindentation using spherical and Berkovich indenters. Comput Methods Biomech Biomed Eng 18(5):492-505

Schwiedrzik JJ, Wolfram U, Zysset PK (2013) A generalized anisotropic quadric yield criterion and its application to bone tissue at multiple length scales. Biomech Model Mechanobiol 12(6):1155-1168

Schwiedrzik J, Raghavan R, Bürki A, LeNader V, Wolfram U, Michler J, Zysset $\mathrm{P}$ (2014) In situ micropillar compression reveals superior strength and ductility but an absence of damage in lamellar bone. Nat Mater 13(7):740-747

Schwiedrzik J, Gross T, Bina M, Pretterklieber M, Zysset P, Pahr D (2016) Experimental validation of a nonlinear $\mu \mathrm{FE}$ model based on cohesive-frictional plasticity for trabecular bone. Int J Numer Methods Biomed Eng. doi:10.1002/cnm.2739
Shi X, Liu XS, Wang X, Guo XE, Niebur GL (2010) Effects of trabecular type and orientation on microdamage susceptibility in trabecular bone. Bone 46(1):1260-1266

Smith IM, Griffiths DV, Margetts L (2013) Programming the finite element method. Wiley, New York

Stölken J, Kinney J (2003) On the importance of geometric nonlinearity in finite-element simulations of trabecular bone failure. Bone 33(4):494-504

Sun X, Hoon Jeon J, Blendell J, Akkus O (2010) Visualization of a phantom post-yield deformation process in cortical bone. J Biomech 43(10):1989-1996

Tai K, Ulm FJ, Ortiz C (2006) Nanogranular origins of the strength of bone. Nano Lett 6(11):2520-2525

Tassani S, Ohman C, Baruffaldi F, Baleani M, Viceconti M (2011) Volume to density relation in adult human bone tissue. J Biomech 44(1):103-108

The MPI Forum (1993) MPI: a message passing interface. In: Proceedings of the conference on high performance networking and computing, pp 878-883. doi:10.1109/SUPERC.1993.1263546

Turner CH, Cowin SC, Rho JY, Ashman RB, Rice JC (1990) The fabric dependence of the orthotropic elastic constants of cancellous bone. J Biomech 23(6):549-561

van Rietbergen B, Weinans H, Huiskes R, Odgaard A (1995) A new method to determine trabecular bone elastic properties and loading using micromechanical finite-element models. J Biomech 28(1):69-81

van Rietbergen B, Odgaard A, Kabel J, Huiskes R (1996) Direct mechanics assessment of elastic symmetries and properties of trabecular bone architecture. J Biomech 29(12):1653-1657

Wang C, Feng L, Jasiuk I (2009) Scale and boundary conditions effects on the apparent elastic moduli of trabecular bone modeled as a periodic cellular solid. J Biomech Eng. doi:10.1115/1.4000192

Wolfram U, Gross T, Pahr DH, Schwiedrzik J, Wilke HJ, Zysset PK (2012) Fabric-based Tsai-Wu yield criteria for vertebral trabecular bone in stress and strain space. J Mech Behav Biomed Mater $15: 218-228$

Zioupos P, Hansen U, Currey JD (2008) Microcracking damage and the fracture process in relation to strain rate in human cortical bone tensile failure. J Biomech 41(14):2932-2939

Zysset PK (2003) A review of morphology elasticity relationships in human trabecular bone: theories and experiments. J Biomech 36(10):1469-1485

Zysset PK, Curnier A (1995) An alternative model for anisotropic elasticity based on fabric tensors. Mech Mater 21(4):243-250

Zysset PK, Curnier A (1996) A 3D damage model for trabecular bone based on fabric tensors. J Biomech 29(12):1549-1558 